\title{
Unilateral loss of trigeminal nerve fibers at the ventral pons
}

\author{
Tabinda Hasan
}

\section{Hasan T. Unilateral loss of trigeminal nerve fibers at the ventral pons. Int J Anat Var. 2019;12(4): 63-65.}

The trigeminal nerve has the largest territory of sensory innervation across the head and neck. It leaves the lateral pons through a large lateral sensory and small medial motor root. Root Entry Zone is an anatomical landmark of great functional hierarchy where variants in nervous tract trajectories and arterial loops, may result in neurovascular conflicts leading to neuralgias. The sensory root holds clinical significance because it innervates sensory receptors of the head and neck.

This case reports a cadaveric brain stem with significant loss/ hypotrophy of left trigerminal nerve fibers. Such forfeit (weather due to congenital hypotrophy or postnatal lesion) can lead to variable degree of sensory loss along the nerve course and the innervation of mastication apparatus, change in of cerebellopontine angel and cistern volumes. The clinical outcomes of missing fibers might be not only hold symptomatic indicators for neurosurgeons but also morphologic benchmarks for biological anthropologists. These incidental findings are noteworthy to understand the causes, intricacies and clinical consequences of such neuroscience dilemmas. The case reported here might have presented with perplexing neurosensory ambiguities while alive and leaves ground of speculation for anatomists and neurologists.

It is fundamental to identify spatial relations between different portions of the nervous tract, (especially while establishing possible vascular contact conflicts) that could explain lesser known Trigerminus syndromes. In future, high spatial resolution imaging would allow the study of each portion of the trigeminal nerve, recognizing with more clarity the anatomical variants or pathological elements.

Key Words: Trigerminal nerve; Trigerminal neuralgia; Absence trigeminal nerve

\section{INTRODUCTION}

A mongst all 12 cranial nerves, the Trigeminal nerve (also called the 5th Nerve) covers the largest area in sensory innervation across the head and neck. The nerve has three sensory nuclei located in the brainstem; namely mesencephalic, chief sensory and spinal nucleus lying inside the midbrain, pons and medulla respectively. The trigeminal nerve leaves the lateral pons through a large sensory and small motor root (rootlets) with a sensory ganglion (called Gassserian ganglion) lying in the Mickel's cave at the apex of the petrous temporal bone [1]. The nerve splits into three divisions; first ophthalmic, second maxillary and third mandibular division that exit the cranium through their respective openings; orbital fissure, foramen rotundum and foramen ovale in order to supply different territories on the. The first two divisions are pure sensory and carry touch, pain and temperature of the facial region, cornea, conjunctival mucosa, paranasal sinuses, and dura of cranial fossa. The third (mandibular portion) is motor to the tempromandibular joint and conveys proprioceptive impulses. It is motor for all the muscles of mastication. Prior to their exit, the three sensory branches of the trigeminal nerve, particularly the ophthalmic and maxillary divisions establish a close relationship with the lateral wall of the cavernous sinus, within the folds of dura-mater.

The location of motor rootlets is relatively closer to the brainstem midline while the sensory rootlets are larger in number and size and are more laterally located. Intricate neuroimaging studies have detailed that among sensory fibres, the first division remains dorso-medial, the second is intermediate while the third division remains ventero-lateral until the formation of the Gassserian ganglion. The Root Entry Zone (REZ) is the point where the roots emerge in the brainstem and it is an anatomical landmark of great functional hierarchy [1,2]. At this pinpoint location, variants in the nervous tract trajectories and the arterial loops, may generate sites of close contact between nerves and pulsating vessels and which may result in neurovascular irritational conflict leading to variable degrees of neuralgias. The sensory root of the Trigeminal nerve is remarkable and holds clinical significance because it innervates peripheral noci-receptors of the head and neck and can be an original site of trigeminal neuralgia [2].

\section{CASE REPORT}

This case reports a cadaveric brain stem exposed during routine head and neck dissection showing left sided hypotrophy/significant loss of the trigeminal nerve root fibers (Figure 1). Since there is no clear cut demarcation visible in the specimen pertaining to identifying separately the sensory or motor roots; it is difficult to comment weather one or both of the above stated were lost.

The nerve of the right side appears perfectly normal in terms of fiber volumetric morphology. When measured by a thread later put to a calibrated vernier scale, the right side nerve showed a circumference of $1.8 \mathrm{~cm}$ while the left side nerve measured $0.7 \mathrm{~cm}(\mathrm{p}<0.05)$. This circumferential difference is statistically significant. To be noted here that the measuring process was repeated by two independent researchers at different occasions while using the same apparatus to avoid observer or measurement bias.

The detailed surgical and radiological anatomy of the trigeminal root is a lesser explored area. The cause of such significant unilateral fiber loss as noted in the specimen is hitherto unreported till date to the best of author's knowledge.

It could be attributed to congenital agenesis or post natal demyelination due to invasive neurological lesion. Absence of fibers can lead to variable degree of sensory loss (hypoesthesia or anesthesia) along the distribution of the trigeminal nerve divisions for the head, face and neck; depending on which fibers are most abundantly devoid at the pontine site. A loss of corneal reflex would have occurred in case the fibers supplying the 'eye

Department of Anatomy, College of Medicine, Princess Nora Bint Abdulrahman University, Riyadh, Saudi Arabia

Correspondence: Dr. Tabinda Hasan, Department of Anatomy, College of Medicine, Princess Nora Bint Abdulrahman University, Riyadh, Saudi Arabia. Telephone +966560211751; E-mail: drtabindahasan@gmail.com

Received: Sep 21, 2019, Accepted: Nov 23, 2019, Published: Dec 02, 2019 


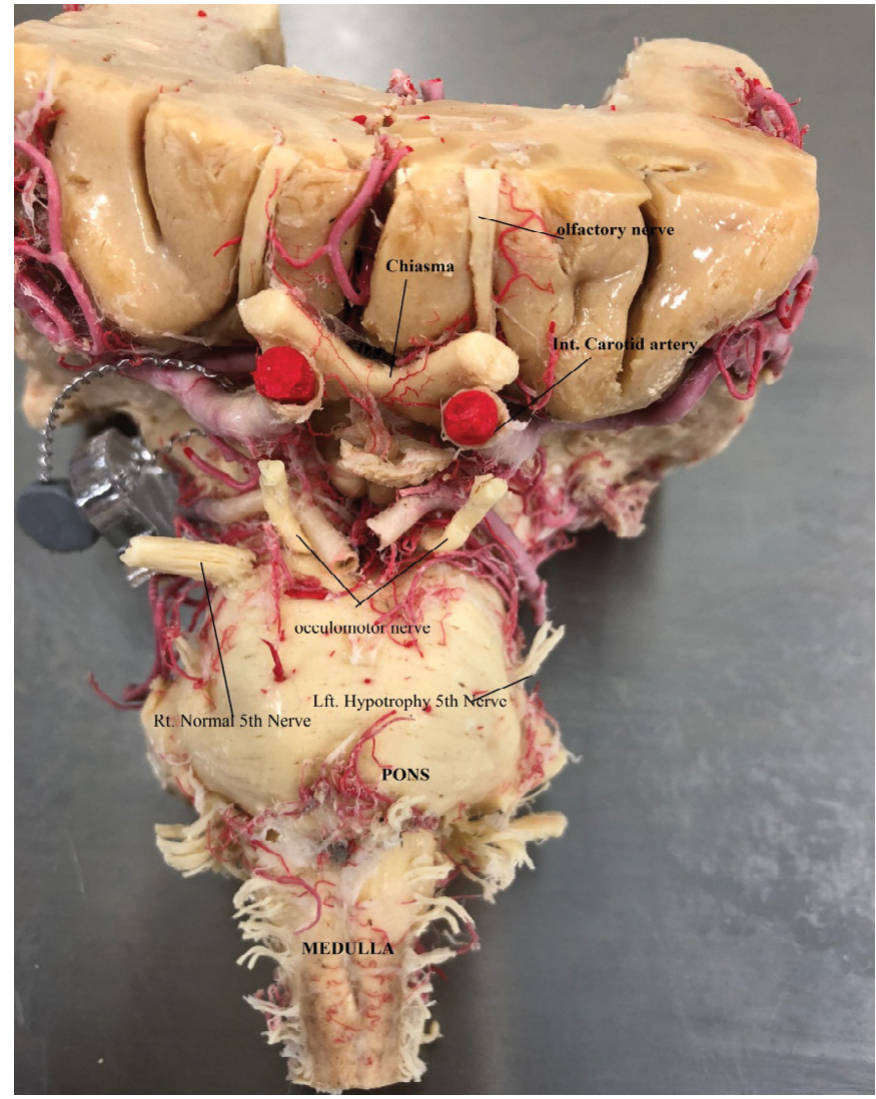

Figure 1) Brainstem showing normal Trigeminal nerve fibers on right side and significant loss of trigeminal nerve fibers on left side of the ventral pons.

area' were lost. The subject would have suffered from proprioceptive loss at the temporomandibular joint (in case the mandibular division was lost) or variable degrees of interruption of the mastication apparatus (in case the motor root was involved).

\section{DISCUSSION}

Neurologists claim that the motor and accessory (aberrant) rootlets have inconsistent courses [3]. They arise from the lateral pons, traverse the' pre-pontine cistern' with the main sensory root, and finally enter into the Meckel's cave. The fate of the motor root is its final merging with the sensory root to form the mandibular nerve to supply lower jaw and teeth.

To our knowledge, very few studies have documented the detailed surgical and radiological anatomy of the trigeminal roots. The intensive work of Tsutsumi and his team in 2018 is commendable in this less explored area [4].

The cause of such significant fiber loss as noted in the specimen (Figure 2) could be attributed to congenital agenesis or post natal demyelination [5]. It might also be possible that the person had previous hemorrhage, infarcts, or tumors in the brainstem, meningitis, traumatic brain injury, hydrocephalus, or lesions in the cavernous sinus that might have eroded the previously healthy nerve roots.

\section{Clinical significance}

Peripheral lesions of sensory portion of trigeminal nerve distal to pontine exit point can produce harrowing ipsilateral pain [6]. This type of facial pain with a seemingly 'normal' patient profile could always be misdiagnosed by the unsuspecting physician as a facial tic condemning the subject to unnecessary psychiatric evaluation or a simple tooth pain sending him off to the dentists.

The location of the lesion or loss always determines the course and gravity of the symptoms [7]. Absence of neurological fibers can lead to variable degree of sensory or motor loss along the distribution of the trigeminal nerve divisions for the head and neck; depending on which fibres are most abundantly devoid at the pontine site; as per the territories marked for the first, second and third divisions. Its loss would definitely have compromised and drastically hindered the ipsilateral sensitive innervation (touch, pain, temperature and propioception) together with the motor innervation of the mastication apparatus.

A loss of the ophthalmic fibers could have led to an absent corneal reflex while a loss of the mandibular fibers could have resulted in unilateral weakness or paralysis of mastication muscles with the jaw deviating towards the paralyzed side or an absent jaw jerk reflex.

Such fiber absence or loss can also alter volume of the cerebellopontine angel cistern since MRI scans show the trigeminal roots of origin significant determinants of such angles [8]. Arachnoid membranes dividing the cistern have been explored in relation to the course of the trigeminal root and neuralgia. So the clinical outcomes of absence might be not only hold symptomatic indicators for neurosurgeons but also morphologic benchmarks for biological anthropologists.

On a contrary note, trigerminal neuralgia originating from vascular compression (particularly the middle and superior cerebellar arteries owing to their physical proximity) holds a well-established etiology and partial or complete absence of fibers might lead to volumetric decompressions and relieve the possibility of such pains [9]. Hence, it might also play some sort of physiologically protective role since the root at the site of neurovascular contact attributes in accentuating neuralgia.

Such incidental findings are noteworthy and deserve being reported to enable neuroscientists understand better the intricacies, causes, consequences and means to avert such clinical dilemmas. The case reported here breeds fertile ground for a representative in 'patients type'; one with possible appearance of symptoms in the facial territory with an established degenerative/ inflammatory disorder of the upper cervical spine or any similar condition involving the head/neck region. The human patient image hypothesized here might yet be in its juvenile stages but still it prompts towards deeper existing morphological lesions that can in future be identified as red flags in neurology clinics in case doctors do encounter such a-typical features among seemingly a-symptomatic patients.

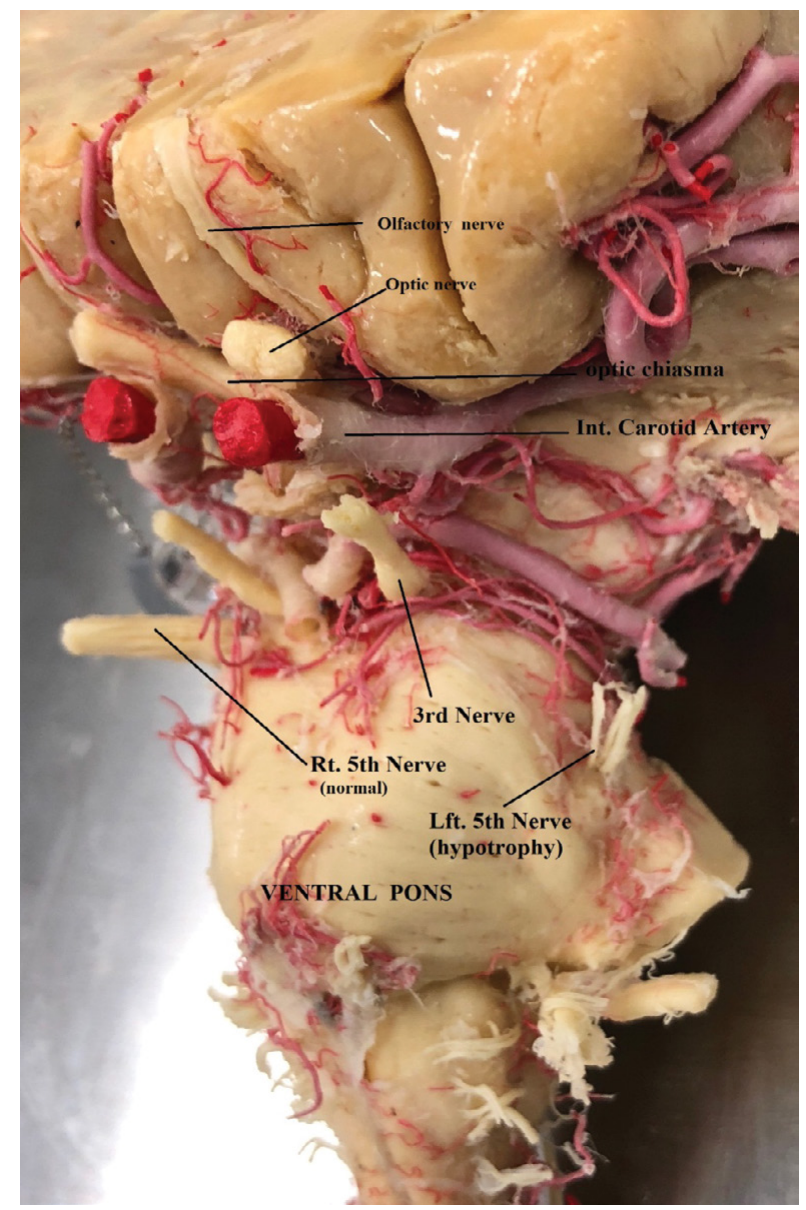

Figure 2A) Brainstem comparing trigeminal nerve fiber volume on right and left side (note the normal nerve of right side as compared to the significant fiber loss on left side). 


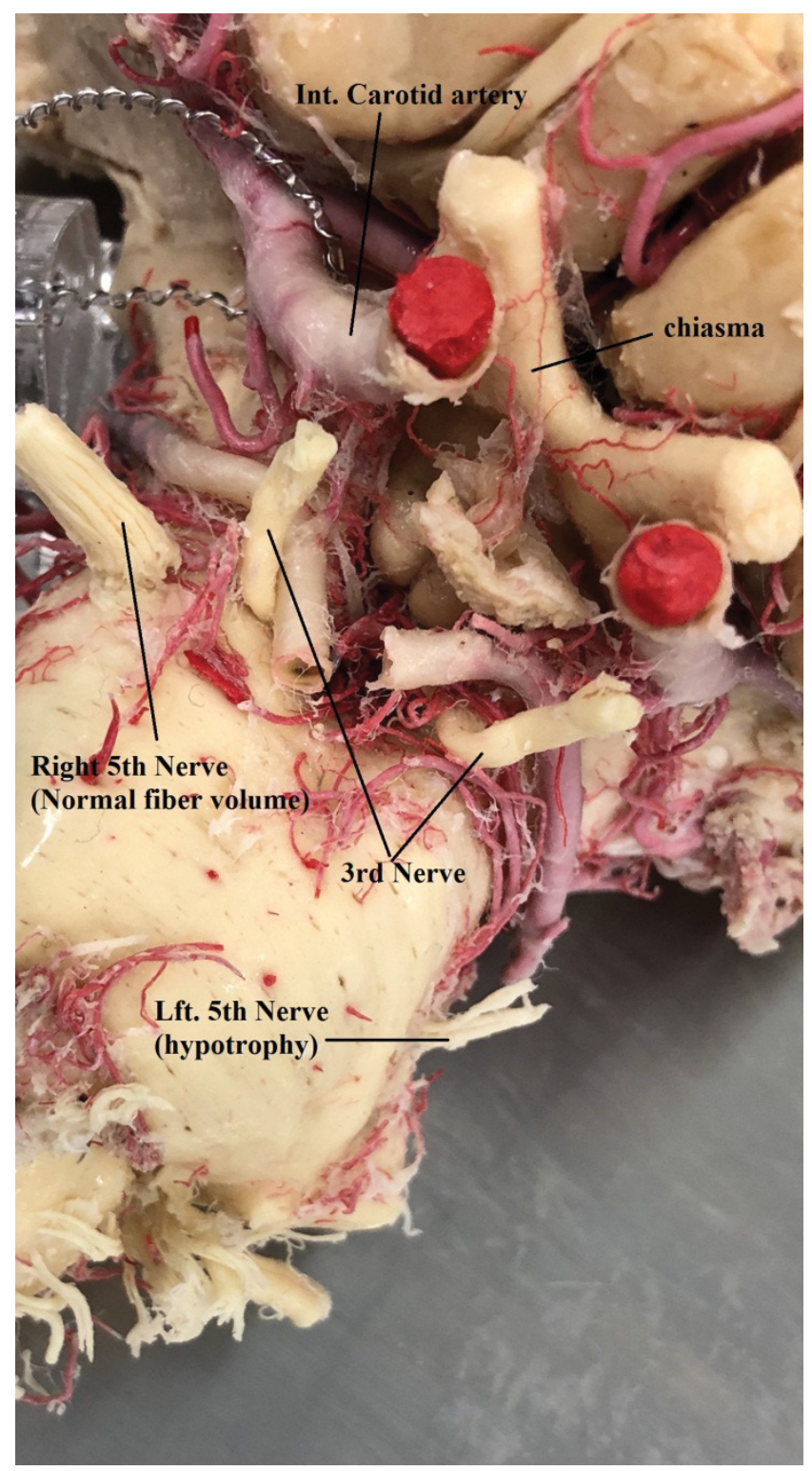

Figure 2B) Brainstem comparing trigeminal nerve fiber volume on right and left side (note the normal nerve of right side as compared to the significant fiber loss on left side).

\section{CONCLUSION}

None the less, the case reported here presents many ambiguities and leaves much ground for speculation to anatomists and neurologists; but it would be a rather welcome field open to further exploration. In some situations it is fundamental to consider the precise anatomical details and identify the spatial relations between the different portions of the nervous tract, especially while trying to establish possible conflicts (at vascular contact points) among the structures. In future, the use of high spatial resolution imaging while we pass from an anatomy of slices to the multi-planar study of these small structures with more information when understanding the 5th Nerve would allow the study of each of the portions of the trigeminal nerve, recognizing with more clarity the anatomical variants and/or pathological elements that could explain the clinical presentation of frequent as well as lesser known Trigerminus syndromes.

\section{REFERENCES}

1. Lee YJ, Moon HC, Tak S, et al. Atrophic Changes and Diffusion Abnormalities of Affected Trigeminal Nerves in Trigeminal Neuralgia Using 7-T MRI. Stereotact Funct Neurosurg. 2019;19:1-7.

2. Dingle A, Zeng W, Ness JP, et al. Strategies for interfacing with the trigeminal nerves in rodents for bioelectric medicine. J Neurosci Methods. 2019;324:108321.

3. Slavin KV, Wess C. Trigeminal branch stimulation for intractable neuropathic pain: technical note. Neuromodulation. 2005 ;8:7-13.

4. Tsutsumi $\mathrm{S}$, Ono $\mathrm{H}$, Yasumoto $\mathrm{Y}$, et al. The trigeminal root: an anatomical study using magnetic resonance imaging. Surg Radiol Anat. 2018;40:1397-1403.

5. Leal PR, Barbier C, Hermier M, et al. Atrophic changes in the trigeminal nerves of patients with trigeminal neuralgia due to neurovascular compression and their association with the severity of compression and clinical outcomes. J Neurosurg. 2014;120:1484-95.

6. Mastronardi L, Caputi F, Rinaldi A, et al. Typical Trigeminal Neuralgia: Comparison of Results between Patients Older and Younger than 65 Operated on with Microvascular Decompression by Retrosigmoid Approach. J Neurol Surg A Cent Eur Neurosurg. 2019.

7. Wolf A, Tyburczy A, Ye JC, et al. The relationship of dose to nerve volume in predicting pain recurrence after stereotactic radiosurgery in trigeminal neuralgia. J Neurosurg. 2018;128:891-6.

8. Tarabay A, Rocca A, Maeder P, et al. Extra-axial cavernoma of the cerebellopontine angle: A case study and review of literature. World Neurosurg. 2019;128:415-21.

9. Gamaleldin OA, Khalifa MH, Donia MM, et al. Role of fused threedimensional time-of-flight MR angiography and 3D T2WI sequences in neurovascular compression. World Neurosurg. 2019. 